\title{
The Berlin Exoplanet Search Telescope. II. Catalog of Variable Stars. III. Census of Variable Stars in a Puppis Field
}

\author{
C. Dreyer ${ }^{1}$, Sz. Csizmadia ${ }^{1}$, R. Titz-Weider ${ }^{1}$, Ph. Eigmüller ${ }^{1,3}$, T. Fruth $^{2}$, J. Cabrera ${ }^{1}$ (D) A. Erikson ${ }^{1}$, and H. Rauer ${ }^{1,4}$ \\ ${ }^{1}$ Institute of Planetary Research, German Aerospace Center, Rutherfordstraße 2, D-12489 Berlin, Germany; claudia.dreyer@dlr.de \\ ${ }^{2}$ German Space Operations Center, German Aerospace Center, Münchener Straße 20, D-82234 Weßling, Germany \\ ${ }^{3}$ Center for Astronomy and Astrophysics, Technische Universität Berlin, Hardenbergstraße 36, D-10623 Berlin, Germany \\ ${ }^{4}$ Institute of Geological Sciences, Malteserstraße 74-100, D-12249 Berlin, Germany \\ Received 2018 July 25; revised 2018 August 26; accepted 2018 August 27; published 2018 October 17
}

\begin{abstract}
The Berlin Exoplanet Search Telescope II (BEST II) is a ground-based, small aperture, wide-angle telescope used to search for stellar light variations in the southern hemisphere. We report the results of a monitoring campaign observing a field in the Puppis constellation in late 2011/early 2012. Light curves were obtained for 130,472 stars, out of which we identify 2169 variables, including 1829 newly discovered, 26 previously known, and 314 suspected variable stars. We determine periods and variability class for two previously known, but only suspected to be, variable stars. For eight individual eclipsing binary stars, including the two previously known but unclassified binaries, the system parameters were derived at the epoch of the observation by modeling the light curves. Eventually, in a catalog for all variable stars, we present coordinates, magnitude, and elements of light variations, e.g., epoch, period, amplitude, type, and light curves. This catalog concludes the BEST/BEST II project.
\end{abstract}

Key words: binaries: eclipsing - catalogs - methods: data analysis - stars: variable: general - techniques: photometric

Supporting material: figure set, machine-readable table

\section{Introduction}

Variable stars provide a variety of opportunities to use them as astrophysical laboratories and to improve our knowledge of stellar astrophysics, interior structure, evolution, contribution to galactic chemistry etc. Binary stars and pulsating variable stars are two fundamentally important groups of celestial objects. Observations of eclipsing binary stars allow us to determine the masses of stars and provide a wealth of information on the density and atmosphere (e.g., Hilditch 2004; Southworth 2012). Pulsating variable stars also provide information about the interiors of stars. They are also extremely useful as distance indicators, making it possible to measure the distance to the clusters and galaxies where they are found (e.g., Hubble 1925; Feast \& Whitelock 2014). The study of stellar variability contributes to detections of transiting extra-solar planets because the light curves of their host stars are characterized by typical low-amplitude periodic events on a scale of a few days. In addition, a light curve may also contain variability from a close star and could lead to false transit candidate identification.

This work is the continuation of previously performed variable star censuses by the Berlin Exoplanet Search Telescope BEST/BEST II project. For the star censuses with BEST from the northern hemisphere, see Karoff et al. (2007), Kabath et al. (2007, 2008, 2009a, 2009b), and Pasternacki et al. (2011) and with BEST II from the southern hemisphere, see Fruth et al. (2012, 2013) and Klagyivik et al. (2013, 2016). Here, we present the variable star observations and classifications from an observing campaign from 2011 November to 2012 April performed by BEST II monitoring a field in the Puppis (Pup) constellation in the southern hemisphere.

This article is structured as follows: at first we give a short overview of the telescope configuration and the observation conditions. In Section 3, we explain the standard procedure for data calibration, photometry, and analysis. The results of the classification are presented in Section 4. In Section 5, we relate our classification with the recently released Gaia data. Section 6 exhibits the modeling results of some specific interesting binary stars. Finally, we summarize our results in Section 7.

\section{Observations}

The Berlin Exoplanet Search Telescope (BEST) is a groundbased photometric search project that was initiated in 2001 by the Institute for Planetary Research of the German Aerospace Agency (DLR). The prime use of the system was to provide robotic observational support to the CoRoT space mission by excluding false positives from the list of transiting planetary candidates prior to and during mission operation (Baglin et al. 2006; Deeg et al. 2009; Csizmadia et al. 2011). In addition, BEST observations should also point out potentially interesting objects for the CoRoT additional science programs, such as variable stars. In various time periods, the regular CoRoT support was not required, and independent surveys were performed. The first observations were carried out in the northern hemisphere at the Thüringer Landessternwarte Tautenburg (TLS), Germany, and later relocated to Observatoire de Haute-Provence (OHP), France, with the BEST telescope (Rauer et al. 2004). Since 2007, BEST II (Kabath et al. 2009a) has operated in the southern hemisphere at the Observatorio Cerro Armazones (OCA), Chile, located at an altitude of $2817 \mathrm{~m}$ above sea level. It has been supported by the Instituto de Astronomia de la Universidad Católica del Norte (UCN) in Antofagasta and the Astronomical Institute, RuhrUniversität Bochum (AIRUB; Rauer et al. 2010).

The small aperture automatic BEST II system consists of a $25 \mathrm{~cm}$ Baker-Ritchey-Chrétien telescope with a focal ratio of $f / 5.0$ on a German-equatorial mount. It is equipped with a CCD 


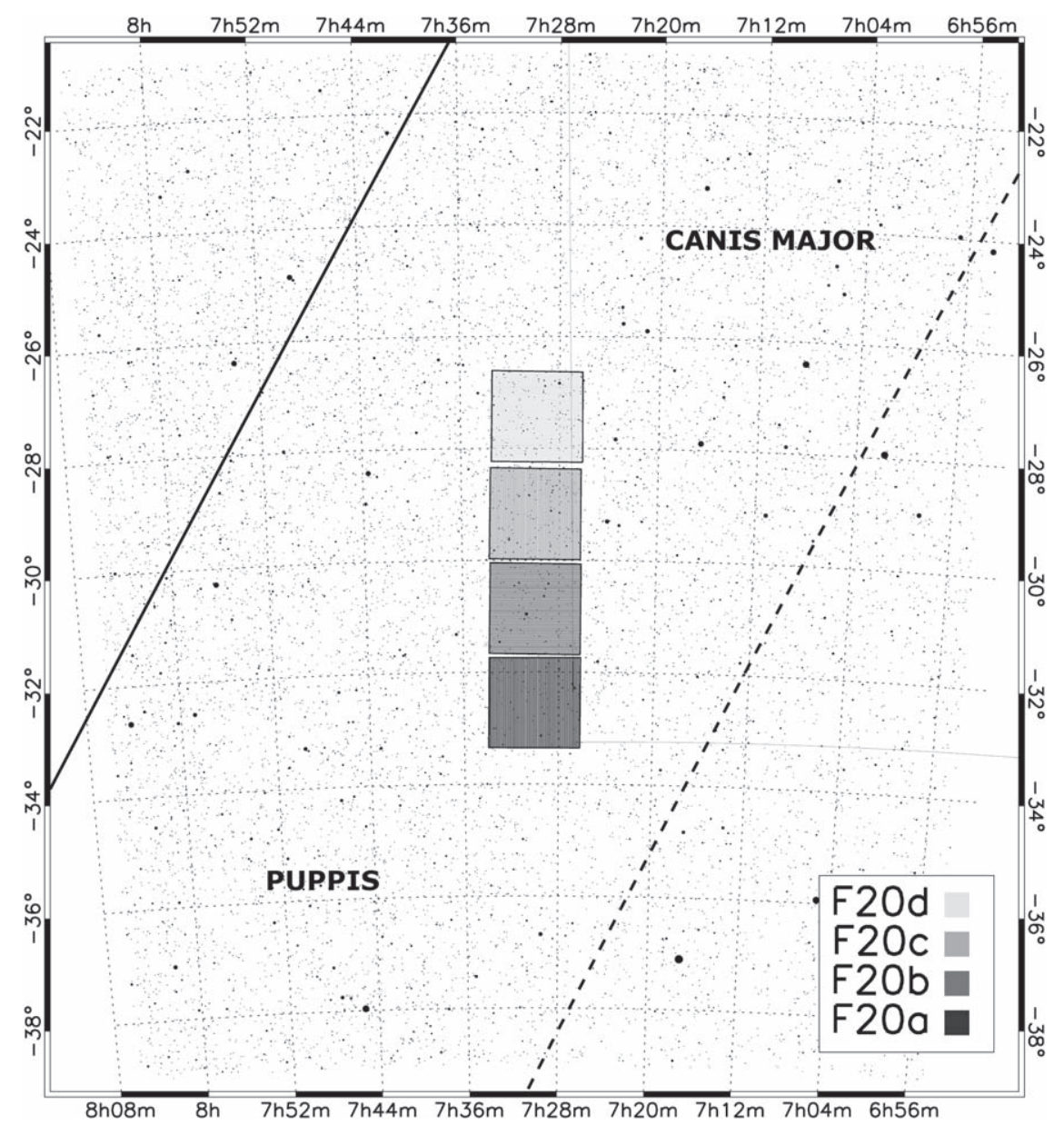

Figure 1. Position of the BEST II F20 subfields in the Puppis constellation indicated in the sky map. It is close to its neighbor constellation Canis Major (thin solid line). The thick solid line represents the Galactic plane and the thick dashed line corresponds to $-10^{\circ}$ Galactic longitude.

camera with $4096 \times 4096$ pixels. The field of view (FoV) of the telescope is $1.7 \times 1^{\circ} 7$, allowing for a large number of stars to be observed simultaneously.

The catalog of variable stars presented here was derived from an observation campaign between 2011 November 9 and 2012 April 6 performed with BEST II monitoring a target field in the Puppis (Pup) constellation (hereafter, field F20) located south of the Galactic plane. Based on lessons learned from former observing campaigns (Pasternacki et al. 2011; Fruth et al. 2012), this BEST II run was optimized for brighter targets to enable an easier radial velocity follow-up process and/or transit spectroscopy to characterize the atmospheric composition of transiting planets. For the target field F20, the exposure time was decreased to $45 \mathrm{~s}$ in order to shift the photometric range by 2 mag toward brighter target stars to 10-13 mag. Four subfields F20a, F20b, F20c, and F20d (see Figure 1) were observed during 61 nights in alternating sequences a-b-c-d (changing after every exposure) so that the decreased number of bright stars was compensated by an FoV that was four times larger. Flat, bias, and dark images were acquired at the beginning and end of each single night observation. All observations were obtained without any filter, i.e., in white light leading to a bandpass limitation by the quantum efficiency of the detector, which peaks in the red at ca. $650 \mathrm{~nm}$ (instrument magnitude $R_{B}$ ) close to the standard Johnson $R$-band at $658 \mathrm{~nm}$. Figure 2 shows the duty cycle of the BEST II observations.
The photometric quality of the acquired data is shown in Figure 3 by the example of subfield F20a. It shows the total noise $\sigma_{\text {tot }}$ and the three major contributors' red noise $\sigma_{\text {red }}$, photon noise $\sigma_{\text {phot }}$, and background noise $\sigma_{\mathrm{bg}}$. The light curves of the brighter stars are dominated by photon noise $\sigma_{\text {phot }}$, but the majority of light curves (fainter stars) are dominated by background noise $\sigma_{\mathrm{bg}}$. The other subfields show similar behavior with negligible differences.

Table 1 summarizes the subfield center coordinates, the time range of the observation, the number of good photometric nights with observations within this range, the number of acquired frames, and the number of total/low-noise light curves for each target field.

\section{Data Reduction}

To calibrate and reduce scientific images from the BEST II telescope, an automated photometric pipeline was applied as outlined in Rauer et al. (2010) and improved by Fruth et al. (2012). It was developed for the BEST project, but is intended to be applicable to any time-series astronomical data set.

To identify potential stellar variability from the huge data set of 130,472 observed stars we applied the method described by Fruth et al. (2012), which is based on the modified Stetson's $j$-variability index (Stetson 1996) according to Zhang et al. (2003). In our work, we set a cutoff limit for potentially variable stars of $j>0.1$ based on experience with previous 


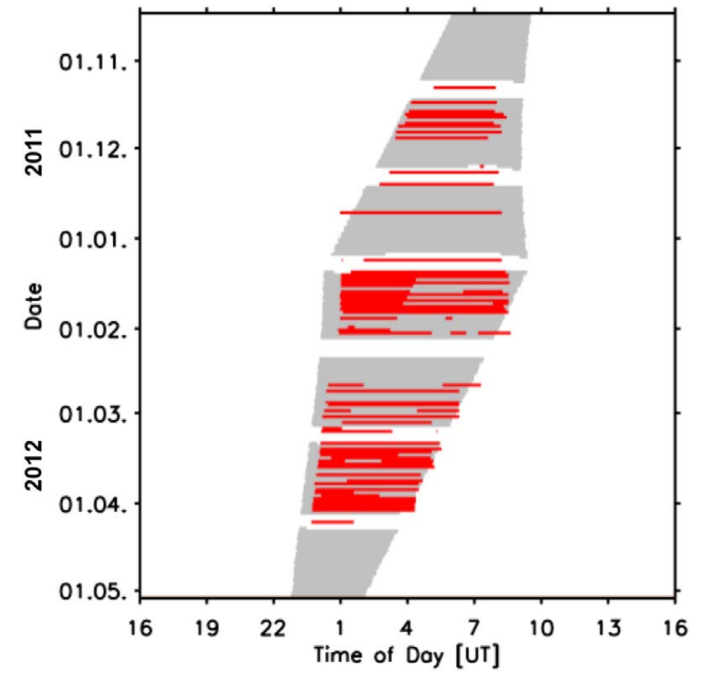

Figure 2. BEST II observation time for the field F20 (red). The gray area specifies optical astronomical visibility of the target field (see Rauer et al. 2008).

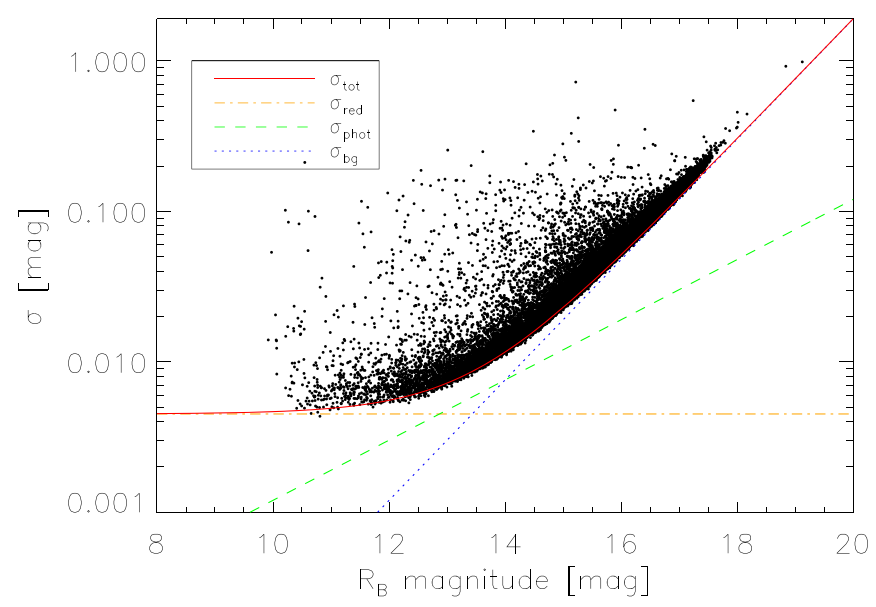

Figure 3. Photometric standard deviation $\sigma$ as a function of instrument magnitude $R_{B}$ of the entire data set of 61 nights for subfield F20a as an example. The solid red line marks the total noise level $\sigma_{\text {tot }}$ of the data. Also shown are the individual contributions of red noise $\sigma_{\text {red }}$ (orange dashed-dotted line), photon noise $\sigma_{\text {phot }}$ (green dashed line), and background noise $\sigma_{\text {bg }}$ (blue dotted line).

BEST II field characterization. The resulting 32,850 light curves were searched for significant frequencies in the range from 0.025 to 50 days by using the Algorithm of Variance (AoV) proposed by Schwarzenberg-Czerny (1996). From that AoV statistic, a modified quality parameter $q$ was calculated giving lower weights to common periodic variability that is encountered in many light curves (see Fruth et al. 2012 for more details). Finally, 2412 light curves with $q \geqslant 10$ were classified by visual inspection. In this process, many false alarms with periods of about 1 day, but no significant presence of stellar variability, were excluded. These are assumed to be mainly artifacts occurring at the sidereal day and caused by the observational duty cycle (Figure 2 ).

\section{Classification}

The classification of the detected variable stars is based on photometric information only, i.e., the shape, the period, and the amplitude of the brightness variation according to the General Catalog of Variable Stars (GCVS) by Samus et al. (2017). A first distinction has to do with the nature of the brightness variations: intrinsic variability is due to physical changes in the star or stellar system. Extrinsic variability is due to the eclipse of one star by another or the effect of stellar rotation.

In our analysis, we used the following subtypes of intrinsic variables: $\delta$ Scuti (DSCT), SX Phoenicise type (SXPHE), $\gamma$ Doradus (GDOR), RR Lyrae (RR), $\delta$ Cephei (DCEP), and Long Period Variable (LPV) stars. The extrinsic variable stars we classified into (semi-)detached eclipsing binary systems or Algol-(EA), the semidetached $\beta$ Lyrae-(EB), the contact binary or W Ursae Majoris-type (EW), the eccentric type (E), and the rotating ellipsoidal variables (ELL). Stars with typical features of star spots are marked as spotted (SP) and periodic PMS stars were marked as young stellar objects (YSOs). In case of doubts, we classified several pulsating-like stars as SP (Poretti et al. 2015). The light curves of the EW and DSCT classes may be indistinguishable with photometric data of a ground-based survey in some low-amplitude cases. For such uncertain classifications, the class of (EW/DSCT) was introduced. Remaining objects showing clear variability but for which no assignment is possible are classified as variable (VAR). This class also includes the semiregular (SR) and miscellaneous (MISC) variables. The classification is highly subjective and depends on the experience of the observer. The agreement with automatic classification is expected to be around $70 \%$ (Fruth et al. 2012). A summary of our classifications containing the counts of a variability class in each subfield is given in Table 2. In total, 1855 variable and 314 suspected variable stars have been detected.

All 2169 detected variable stars are listed in a catalog containing coordinates $\alpha, \delta$ given for epoch $\mathrm{J} 2000.0$ and matched to the 2MASS catalog, 2MASS identification number 2MASS ID, instrument magnitude $R_{B}$, epoch $T_{0}$, period $P$, amplitude $A, j$-index, variability type, and other names where applicable. Stars contaminated with neighboring stars in the aperture, i.e., having overlapping point-spread functions, are marked by a c-flag, already known variables by a k flag, and suspected variables by an s-flag. Table 3 shows an excerpt of the catalog for guidance regarding its form and content. Some textbook light curves are shown in Figure 4. The complete catalog and figure set of all light curves are available online.

\subsection{Known Variable Stars}

The stars observed with BEST II have been cross-checked with the GCVS by Samus et al. (2017) and the international variable star index (VSX; Watson et al. 2015). Within the observed target field F20, a total of 26 previously known variable stars could be found. Table 4 shows the comparison of the classifications and the BEST II light curves are presented in Figure 5.

In general, we confirm the previously determined periods and the stellar variability classes. For two known but only suspected variable stars, NSV 3636 (F20b_27380) (Strohmeier et al. 1964) and NSV 3640 (F20b_30270) (Strohmeier \& Knigge 1975), we determine the type of variability as a contact binary system (EW) with an orbital period of $0.56308 \pm$ 0.00006 days and a type of (semi-)detached eclipsing binary system (EA) with a period of $3.912 \pm 0.007$ days, respectively. For ASAS J073043-3005.6 (F20b_33375), we confirm the period of $0.779 \pm 0.002$ days but not the proposed classes of first-tone classical Cepheid (DCEP-FO), contact binary (EC) or semidetached eclipsing binary (ESD) as suggested by 
Table 1

BEST II Target Field F20 Information

\begin{tabular}{|c|c|c|c|c|c|c|c|}
\hline \multirow{2}{*}{ FIELD } & \multicolumn{2}{|c|}{ Center Coordinates (J2000.0) } & \multirow{2}{*}{ Season } & \multirow{2}{*}{ Nights } & \multirow{2}{*}{ Frames } & \multicolumn{2}{|c|}{ Stars } \\
\hline & $\alpha$ & $\delta$ & & & & Total & $\sigma \leqslant 0.01^{m}$ \\
\hline F20a & $07^{\mathrm{h}} 30^{\mathrm{m}} 00^{\mathrm{s}}$ & $-32^{\circ} 33^{\prime} 00^{\prime \prime}$ & $09 / 11 / 2011-06 / 04 / 2012$ & 61 & 2001 & 31,174 & 1608 \\
\hline $\mathrm{F} 20 \mathrm{~b}$ & $07^{\mathrm{h}} 30^{\mathrm{m}} 00^{\mathrm{s}}$ & $-30^{\circ} 51^{\prime} 00^{\prime \prime}$ & $09 / 11 / 2011-06 / 04 / 2012$ & 61 & 1980 & 34,150 & 1887 \\
\hline $\mathrm{F} 20 \mathrm{c}$ & $07^{\mathrm{h}} 30^{\mathrm{m}} 00^{\mathrm{s}}$ & $-29^{\circ} 09^{\prime} 00^{\prime \prime}$ & $09 / 11 / 2011-06 / 04 / 2012$ & 61 & 1958 & 34,126 & 2004 \\
\hline F20d & $07^{\mathrm{h}} 30^{\mathrm{m}} 00^{\mathrm{s}}$ & $-27^{\circ} 27^{\prime} 00^{\prime \prime}$ & $09 / 11 / 2011-06 / 04 / 2012$ & 61 & 2010 & 31,022 & 1096 \\
\hline
\end{tabular}

Table 2

Statistical Overview of All Detected Variable Stars

\begin{tabular}{|c|c|c|c|c|c|c|c|c|c|c|}
\hline \multirow{4}{*}{$\begin{array}{l}\text { Total } \\
\text { Stars with } j>0.1 \\
\text { Stars with } q>10\end{array}$} & \multicolumn{2}{|c|}{ F20a } & \multicolumn{2}{|c|}{ F20b } & \multicolumn{2}{|c|}{ F20c } & \multicolumn{2}{|c|}{ F20d } & \multicolumn{2}{|c|}{ Total F20 } \\
\hline & \multicolumn{2}{|c|}{31,174} & \multicolumn{2}{|c|}{34,150} & \multicolumn{2}{|c|}{34,126} & \multicolumn{2}{|c|}{31,022} & \multicolumn{2}{|c|}{130,472} \\
\hline & \multicolumn{2}{|c|}{$7004^{(23 \%)}$} & \multicolumn{2}{|c|}{$8769^{(26 \%)}$} & \multicolumn{2}{|c|}{$8481^{(25 \%)}$} & \multicolumn{2}{|c|}{$8596^{(28 \%)}$} & \multicolumn{2}{|c|}{$32,850^{(25 \%)}$} \\
\hline & \multicolumn{2}{|c|}{$550^{(1.8 \%)}$} & \multicolumn{2}{|c|}{$635^{(1.9 \%)}$} & \multicolumn{2}{|c|}{$659^{(1.9 \%)}$} & \multicolumn{2}{|c|}{$568^{(1.8 \%)}$} & \multicolumn{2}{|c|}{$2412^{(1.9 \%)}$} \\
\hline \multicolumn{11}{|l|}{ Intrinsic } \\
\hline DCEP & 44 & (3) & 55 & (12) & 37 & (5) & 16 & (2) & 152 & (22) \\
\hline DSCT & 105 & (21) & 85 & (30) & 119 & (23) & 56 & (15) & 365 & (89) \\
\hline GDOR & 1 & (0) & 0 & (0) & 0 & $(0)$ & 0 & (0) & 1 & (0) \\
\hline LPV & 16 & (1) & 21 & (2) & 16 & (0) & 18 & (1) & 71 & (4) \\
\hline RR & 57 & (1) & 49 & (14) & 47 & (8) & 36 & (14) & 189 & (37) \\
\hline SXPHE & 2 & (1) & 7 & $(0)$ & 0 & (0) & 1 & $(0)$ & 10 & (1) \\
\hline \multicolumn{11}{|l|}{ Extrinsic } \\
\hline EA & 69 & (6) & 90 & (14) & 107 & (16) & 76 & (9) & 342 & (45) \\
\hline EB & 29 & (1) & 17 & (3) & 23 & (2) & 17 & (2) & 86 & (8) \\
\hline EW & 58 & (1) & 69 & (12) & 58 & (6) & 48 & (2) & 233 & (21) \\
\hline EW/DSCT & 4 & (1) & 7 & (2) & 6 & (2) & 26 & (5) & 43 & (10) \\
\hline E & 0 & (0) & 0 & (0) & 5 & (1) & 1 & (1) & 6 & (2) \\
\hline ELL & 6 & (0) & 9 & (3) & 16 & (8) & 2 & (2) & 33 & (13) \\
\hline SP & 28 & (9) & 29 & (4) & 64 & (9) & 41 & (23) & 162 & (45) \\
\hline YSO & 4 & (1) & 0 & (0) & 1 & (1) & 0 & (1) & 5 & (3) \\
\hline VAR & 36 & (1) & 41 & (6) & 27 & (5) & 53 & (2) & 157 & (14) \\
\hline \multirow[t]{2}{*}{ Total } & 459 & $(47)$ & 479 & (102) & 526 & (86) & 391 & (79) & 1855 & (314) \\
\hline & $1.5 \%$ & $0.15 \%$ & $1.4 \%$ & $0.29 \%$ & $1.5 \%$ & $0.25 \%$ & $1.3 \%$ & $0.26 \%$ & $1.4 \%$ & $0.24 \%$ \\
\hline
\end{tabular}

Note. Given are the numbers of newly detected and already known variable stars for each subfield per variability class. Percentage values give the relative fraction compared to the total count whereas integers in brackets state the number of suspected variables.

Pojmanski (2002). Clearly showing multiple aliases, the shape of the light curve does not support the classification as an eclipsing binary. We have classified it as RR Lyrae instead of a Cepheid because of a period less than 1 day following the GSCV variable type classification. The already known semiregular variables were classified as VAR in our BEST II catalog as we do not further distinguish. For a few variable stars, we are not able to give a period because of our short observation baseline, which resolves periods less than 50 days only, or unclear variability. The GCVS catalog lists them as Mira-type stars (M). We classify them as LPV-the parent class of that type of variable or as VAR.

\subsection{Newly Detected Variable Stars}

In Field F20, we detected 1829 new variable stars. In the catalog of BEST II target field F20, the most common types are of short period pulsators (DSCT, RR) with 554 objects and eclipsing binaries (EA, EB, EW) with 661 objects. This is approximately two-thirds of all detected variable stars in that field.
Finally, we compared the overall variability fraction of the F20 field to similar ground-based photometric surveys (see Table 5). The percentage of variable stars in the entire F20 data set of 130,472 stars amounts to $1.66 \%$ and is of the same order as in other photometric surveys, e.g., ASAS-2 with $2.7 \%$ and HAT 199 with $1.65 \%$. Table 5 also gives a summary of the total yields of the BEST/BEST II search for light variations, which is now completed.

\section{Color-Magnitude Diagram (CDM)}

Sometimes it is difficult to distinguish between different classes of variable stars and/or the classification is uncertain just by the light-curve shape, periods, and amplitudes. Additional astrometric and color information can help clarify and identify potential systematic problems by producing a CDM. Therein each variability class is associated with a welldefined position.

The Gaia mission (Gaia Collaboration et al. 2016), as an enormous stellar census, delivers us basic astronomical data of unprecedented scope, accuracy, and completeness. We produce CDMs of our BEST II field 20 with the help of the recently 
Table 3

Catalog of Variable Stars Detected in BEST II Field F20 (Excerpt)

\begin{tabular}{|c|c|c|c|c|c|c|c|c|c|c|c|}
\hline BEST II ID & Flag & 2MASS ID & $\alpha(\mathrm{J} 2000.0)$ & $\delta(\mathrm{J} 2000.0)$ & $R_{B}(\mathrm{mag})$ & $T_{0}(\mathrm{rHJD})$ & $P$ (day) & $A$ (mag) & $j$-index & Type & Other Names \\
\hline \multicolumn{12}{|c|}{ F20a } \\
\hline F20a_00022 & c & $07292719-3321460$ & $07^{\mathrm{h}} 29^{\mathrm{m}} 27^{\mathrm{s}} .2$ & $-33^{\circ} 21^{\prime} 46^{\prime \prime} 0$ & 14.09 & 75.779 & $0.09208 \pm 0.00002$ & $0.09 \pm 0.02$ & 1.85 & DSCT & \\
\hline F20a_00116 & & $07270084-3321238$ & $07^{\mathrm{h}} 27^{\mathrm{m}} 00^{\mathrm{s}} .8$ & $-33^{\circ} 21^{\prime} 23^{\prime \prime} 8$ & 13.53 & 75.772 & $0.12409 \pm 0.00004$ & $0.02 \pm 0.02$ & 0.936 & DSCT & \\
\hline F20a_00123 & $\mathrm{s}$ & $07303529-3321151$ & $07^{\mathrm{h}} 30^{\mathrm{m}} 35^{\mathrm{s}} \cdot 3$ & $-33^{\circ} 21^{\prime} 15^{\prime \prime} 1$ & 12.28 & 75.741 & $0.035386 \pm 0.000002$ & $0.01 \pm 0.01$ & 0.942 & DSCT & \\
\hline F20a_00185 & $\mathrm{s}$ & $07262353-3321067$ & $07^{\mathrm{h}} 26^{\mathrm{m}} 23^{\mathrm{s}} .5$ & $-33^{\circ} 21^{\prime} 06^{\prime \prime} 8$ & 13.23 & 83.286 & $1.113 \pm 0.002$ & $0.13 \pm 0.03$ & 3.72 & EA & \\
\hline F20a_00221 & & $07334829-3320207$ & $07^{\mathrm{h}} 33^{\mathrm{m}} 48^{\mathrm{s}} .3$ & $-33^{\circ} 20^{\prime} 20^{\prime \prime} 8$ & 14.60 & 80.179 & $0.925 \pm 0.002$ & $0.09 \pm 0.05$ & 1.11 & GDOR & \\
\hline F20a_00234 & & $07330344-3320285$ & $07^{\mathrm{h}} 33^{\mathrm{m}} 03^{\mathrm{s}} \cdot 4$ & $-33^{\circ} 20^{\prime} 28^{\prime \prime} 7$ & 15.75 & 80.382 & $0.8455 \pm 0.0007$ & $0.30 \pm 0.06$ & 1.29 & EW & \\
\hline \multicolumn{12}{|c|}{$\mathrm{F} 20 \mathrm{~b}$} \\
\hline F20b_00200 & $\mathrm{c}$ & $07333340-3139432$ & $07^{\mathrm{h}} 33^{\mathrm{m}} 33^{\mathrm{s}} \cdot 4$ & $-31^{\circ} 39^{\prime} 43^{\prime \prime} 1$ & 14.97 & 84.891 & $0.3827 \pm 0.0001$ & $0.36 \pm 0.04$ & 3.23 & EW & \\
\hline F20b_00215 & cs & $07314278-3140071$ & $07^{\mathrm{h}} 31^{\mathrm{m}} 42^{\mathrm{s}} .8$ & $-31^{\circ} 40^{\prime} 07^{\prime \prime} 1$ & 14.22 & 84.867 & $0.07747 \pm 0.00002$ & $0.02 \pm 0.03$ & 0.440 & DSCT & \\
\hline F20b_00258 & cs & $07305675-3140047$ & $07^{\mathrm{h}} 30^{\mathrm{m}} 56^{\mathrm{s}} .8$ & $-31^{\circ} 40^{\prime} 04^{\prime \prime} 7$ & 11.91 & 84.902 & $0.4381 \pm 0.0004$ & $0.11 \pm 0.02$ & 7.66 & $\mathrm{RR}$ & \\
\hline $\begin{array}{l}\text { F20b_00259 } \\
\vdots\end{array}$ & $\mathrm{c}$ & $07305736-3140034$ & $07^{\mathrm{h}} 30^{\mathrm{m}} 57^{\mathrm{s}} .3$ & $-31^{\circ} 40^{\prime} 03^{\prime \prime} 5$ & 12.76 & 84.952 & $0.4378 \pm 0.0009$ & $0.09 \pm 0.04$ & 4.03 & $\mathrm{RR}$ & \\
\hline F20b_27380 & $\mathrm{k}$ & $07323060-3023200$ & $07^{\mathrm{h}} 32^{\mathrm{m}} 30^{\mathrm{s}} \cdot 6$ & $-30^{\circ} 23^{\prime} 20^{\prime \prime} 0$ & 11.50 & 75.830 & $0.56308 \pm 0.00006$ & $0.90 \pm 0.03$ & 50.3 & EW & NSV 3636 \\
\hline F20b_30270 & $\mathrm{k}$ & $07323023-3014599$ & $07^{\mathrm{h}} 32^{\mathrm{m}} 30^{\mathrm{s}} \cdot 2$ & $-30^{\circ} 14^{\prime} 59^{\prime \prime} 8$ & 10.66 & 83.035 & $3.912 \pm 0.007$ & $1.4 \pm 0.2$ & 32.5 & EA & NSV 3640 \\
\hline \multicolumn{12}{|c|}{ F20c } \\
\hline F20c_00021 & & $07302379-2959153$ & $07^{\mathrm{h}} 30^{\mathrm{m}} 23.8$ & $-29^{\circ} 59^{\prime} 15^{\prime \prime} 3$ & 15.06 & 90.795 & $8.56 \pm 0.06$ & $0.32 \pm 0.06$ & 0.763 & EA & \\
\hline F20c_00133 & c & $07324242-2958304$ & $07^{\mathrm{h}} 32^{\mathrm{m}} 42^{\mathrm{s}} .4$ & $-29^{\circ} 58^{\prime} 30^{\prime \prime} 2$ & 12.46 & 84.873 & $0.08876 \pm 0.00003$ & $0.02 \pm 0.02$ & 1.21 & DSCT & \\
\hline F20c_00421 & & $07284237-2958038$ & $07^{\mathrm{h}} 28^{\mathrm{m}} 42 \mathrm{~s} .4$ & $-29^{\circ} 58^{\prime} 04^{\prime \prime} 0$ & 14.21 & 159.161 & $21.8 \pm 0.3$ & $0.39 \pm 0.03$ & 4.48 & EB & \\
\hline F20c_00433 & & 07331610-2957148 & $07^{\mathrm{h}} 33^{\mathrm{m}} 16^{\mathrm{s}} .1$ & $-29^{\circ} 57^{\prime} 14^{\prime \prime} 9$ & 11.86 & $\ldots$ & $\ldots$ & $\ldots$ & 13.6 & VAR & \\
\hline F20c_00457 & $\mathrm{s}$ & $07313726-2957330$ & $07^{\mathrm{h}} 31^{\mathrm{m}} 37^{\mathrm{s}} .3$ & $-29^{\circ} 57^{\prime} 32^{\prime \prime} 8$ & 12.42 & 84.993 & $0.16863 \pm 0.00009$ & $0.02 \pm 0.02$ & 1.20 & DSCT & \\
\hline F20c_00518 & & $07304424-2957278$ & $07^{\mathrm{h}} 30^{\mathrm{m}} 44^{\mathrm{s}} \cdot 2$ & $-29^{\circ} 57^{\prime} 27^{\prime \prime} 8$ & 14.50 & 84.927 & $0.13222 \pm 0.00005$ & $0.04 \pm 0.03$ & 0.415 & DSCT & \\
\hline \multicolumn{12}{|c|}{ F20d } \\
\hline F20d_00027 & & $07285717-2814566$ & $07^{\mathrm{h}} 28^{\mathrm{m}} 57^{\mathrm{s}} \cdot 2$ & $-28^{\circ} 14^{\prime} 56^{\prime \prime} 6$ & 15.08 & 85.213 & $0.6971 \pm 0.0009$ & $0.06 \pm 0.04$ & 0.372 & EW & \\
\hline F20d_00102 & & $07311699-2814309$ & $07^{\mathrm{h}} 31^{\mathrm{m}} 17^{\mathrm{s}} .0$ & $-28^{\circ} 14^{\prime} 31^{\prime \prime} 0$ & 14.47 & 85.073 & $0.3887 \pm 0.0005$ & $0.06 \pm 0.03$ & 0.937 & SP & \\
\hline F20d_00146 & & $07293598-2814338$ & $07^{\mathrm{h}} 29^{\mathrm{m}} 36^{\mathrm{s}} .0$ & $-28^{\circ} 14^{\prime} 34^{\prime \prime} 0$ & 14.79 & 147.795 & $20.5 \pm 0.6$ & $0.18 \pm 0.03$ & 2.33 & DCEP & \\
\hline F20d_00178 & & $07332303-2813562$ & $07^{\mathrm{h}} 33^{\mathrm{m}} 23^{\mathrm{s}} .0$ & $-28^{\circ} 13^{\prime} 56^{\prime \prime} 2$ & 12.75 & 85.356 & $2.472 \pm 0.006$ & $0.16 \pm 0.03$ & 2.77 & EA & \\
\hline F20d_00359 & $\mathrm{s}$ & $07280939-2814056$ & $07^{\mathrm{h}} 28^{\mathrm{m}} 09^{\mathrm{s}} .4$ & $-28^{\circ} 14^{\prime} 05^{\prime \prime} 6$ & 14.44 & 87.510 & $1.506 \pm 0.004$ & $0.05 \pm 0.02$ & 0.319 & EB & \\
\hline F20d_00373 & & $07310476-2813489$ & $07^{\mathrm{h}} 31^{\mathrm{m}} 04^{\mathrm{s}} .8$ & $-28^{\circ} 13^{\prime} 49^{\prime \prime} 3$ & 12.35 & $\ldots$ & $\ldots$ & $\ldots$ & 4.52 & LPV & \\
\hline
\end{tabular}

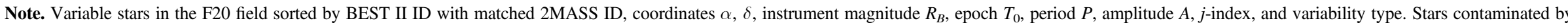

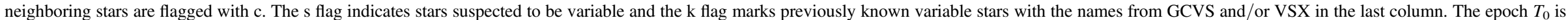
given in reduced Julian date (rHJD) in respect to $T=2455,800.0$

(This table is available in its entirety in machine-readable form.) 

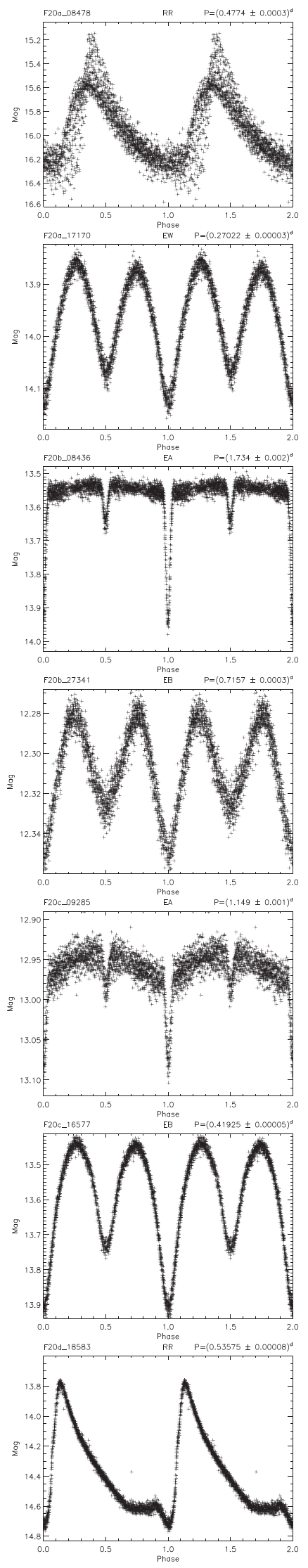
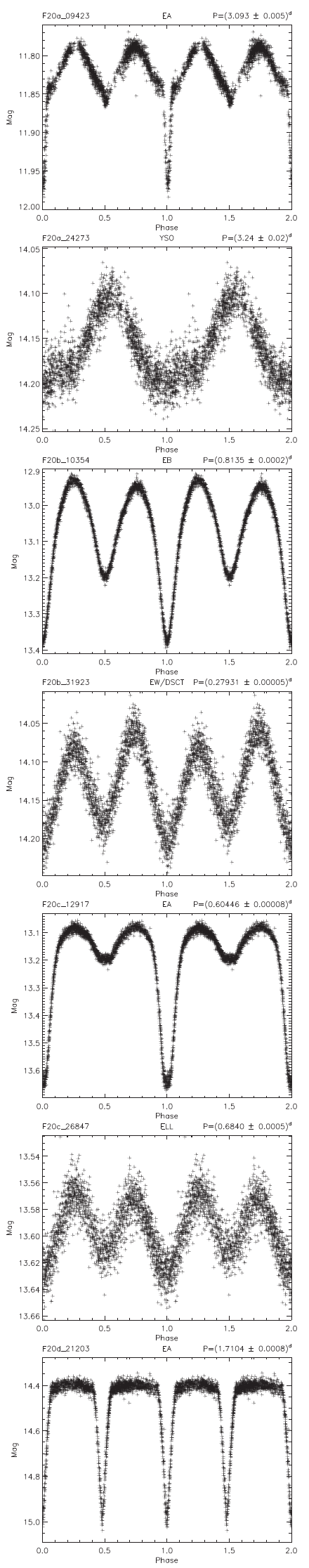
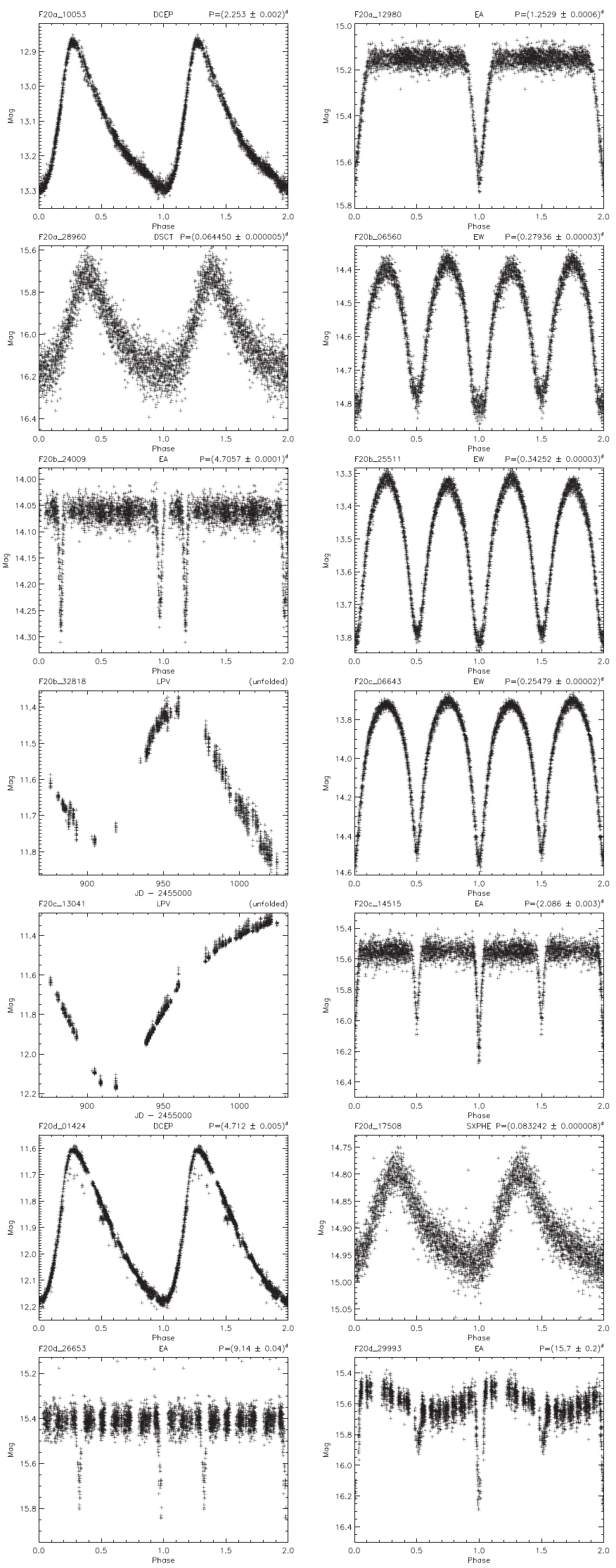
figures. All 2169 light curves are available in the figure set.

(The complete figure set (2169 images) is available.) 
Table 4

Known Variable Stars in the F20 Field

\begin{tabular}{|c|c|c|c|c|c|c|c|}
\hline \multicolumn{2}{|c|}{ Identifier } & \multirow{2}{*}{$\begin{array}{c}R_{B} \\
(\mathrm{mag})\end{array}$} & \multicolumn{2}{|c|}{ Period $P$ (days) } & \multicolumn{2}{|c|}{ Classification } & \multirow{2}{*}{ References } \\
\hline BEST II & Ref. & & BEST II & Ref. & BEST II & Ref. & \\
\hline \multicolumn{8}{|c|}{$F 20 a$} \\
\hline F20a_05669 & ASAS J073332-3300.2 & 9.99 & $\ldots$ & 44.8 & VAR & MISC & Pojmanski (2002), Skiff (2004) \\
\hline F20a_15831 & ASAS J073039-3231.0 & 10.59 & $\ldots$ & 74.8 & LPV & MISC & Pojmanski (2002), Skiff (2004) \\
\hline F20a_19297 & ASAS J073035-3221.5 & 10.44 & $\ldots$ & 70.3 & LPV & MISC & Pojmanski (2002) \\
\hline F20a_19891 & ASAS J073206-3219.7 & 12.38 & $0.47187(5)$ & 0.471874 & EW & EC|ESD & Pojmanski (2002) \\
\hline F20a_20477 & ASAS J073004-3218.3 & 10.59 & $\cdots$ & 98.3 & LPV & MISC & Pojmanski (2002) \\
\hline \multicolumn{8}{|c|}{$F 20 b$} \\
\hline F20b_02668 & ASAS J073102-3130.8 & 12.89 & $0.39263(4)$ & 0.392639 & EW & EC|ESD & Pojmanski (2002) \\
\hline F20b_10156 & ASAS J073206-3108.1 & 13.20 & $1.7825(7)$ & 1.78254 & EA & $\mathrm{ESD}|\mathrm{EC}| \mathrm{ED}$ & Pojmanski (2002) \\
\hline F20b_12203 & ASAS J072931-3103.2 & 10.70 & $\ldots$ & 83.1 & LPV & MISC & Pojmanski (2002) \\
\hline F20b_21665 & ASAS J072830-3039.3 & 11.63 & $3.306(4)$ & 3.3063 & DCEP & DCEPS & Pojmanski (2002), Mel'nik et al. (2015) \\
\hline F20b_24806 & ASAS J072907-3030.8 & 10.17 & .. & 77.3 & VAR & MISC & Pojmanski (2002) \\
\hline F20b_27380 & NSV 3636 & 11.50 & $0.56308(6)$ & $\cdots$ & EW & Suspected & Strohmeier et al. (1964) \\
\hline F20b_30270 & NSV 3640 & 10.66 & $3.912(7)$ & $\cdots$ & EA & Suspected & Strohmeier \& Knigge (1975) \\
\hline F20b_33375 & ASAS J073043-3005.6 & 10.23 & $0.779(2)$ & 0.779260 & $\mathrm{RR}$ & DCEP-FO|EC|ESD & Pojmanski (2002) \\
\hline \multicolumn{8}{|c|}{$F 20 c$} \\
\hline F20c_13759 & ASAS J072840-2920.6 & 12.32 & $2.702(1)$ & 2.7017 & DCEP & DCEP & Pojmanski (2002), Mel'nik et al. (2015) \\
\hline F20c_15448 & V0448 Pup & 10.37 & $\ldots$ & $\cdots$ & VAR & SR: & Pojmanski (2002), Skiff (2004) \\
\hline F20c_16945 & ASAS J073328-2911.5 & 11.56 & $0.6704(1)$ & 0.6704 & EA & $\mathrm{ED} \mid \mathrm{ESD}$ & Pojmanski (2002) \\
\hline F20c_19594 & V0452 Pup & 10.43 & .. & .. & VAR & SR: & Skiff (2014), Takamizawa (2000) \\
\hline F20c_22898 & ASAS J073239-2856.1 & 11.66 & 3.061(3) & 3.060854 & EA & ED & Pojmanski (2002) \\
\hline F20c_26155 & ASAS J072837-2847.6 & 10.03 & & 112.4 & VAR & MISC & Pojmanski (2002), Skiff (2004) \\
\hline \multicolumn{8}{|c|}{ F2Od } \\
\hline F20d_01383 & ASAS J073113-2811.0 & 16.13 & $4.71(8)$ & 4.7092 & DCEP & DCEP & Pojmanski (2002), Pietrukowicz et al. (2013) \\
\hline F20d_07449 & BY CMa & 12.55 & $3.185(2)$ & 3.18493 & DCEP & DCEP & Pojmanski (2002) \\
\hline F20d_27979 & ASAS J072910-2653.3 & 10.39 & $\ldots$ & 313. & LPV & M & Pojmanski (2002), Skiff (2004), Henden et al. (2016) \\
\hline F20d_29374 & BO Pup & 11.14 & $\cdots$ & 615.00000 & LPV & M & Pojmanski (2002), van Hoof (1941) \\
\hline F20d_29927 & ASAS J072618-2643.4 & 9.96 & $\cdots$ & 41.22 & VAR & MISC & Pojmanski (2002), Skiff (2004) \\
\hline F20d_30252 & ASAS J073109-2641.2 & 11.01 & $\ldots$ & 236. & VAR & MISC & Pojmanski (2002) \\
\hline F20d_30704 & V0451 Pup & 10.57 & $\ldots$ & $\ldots$ & VAR & SR: & Pojmanski (2002), Skiff (2004), Takamizawa (2000) \\
\hline
\end{tabular}

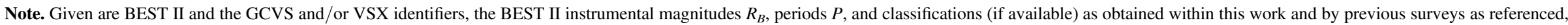
respectively. 

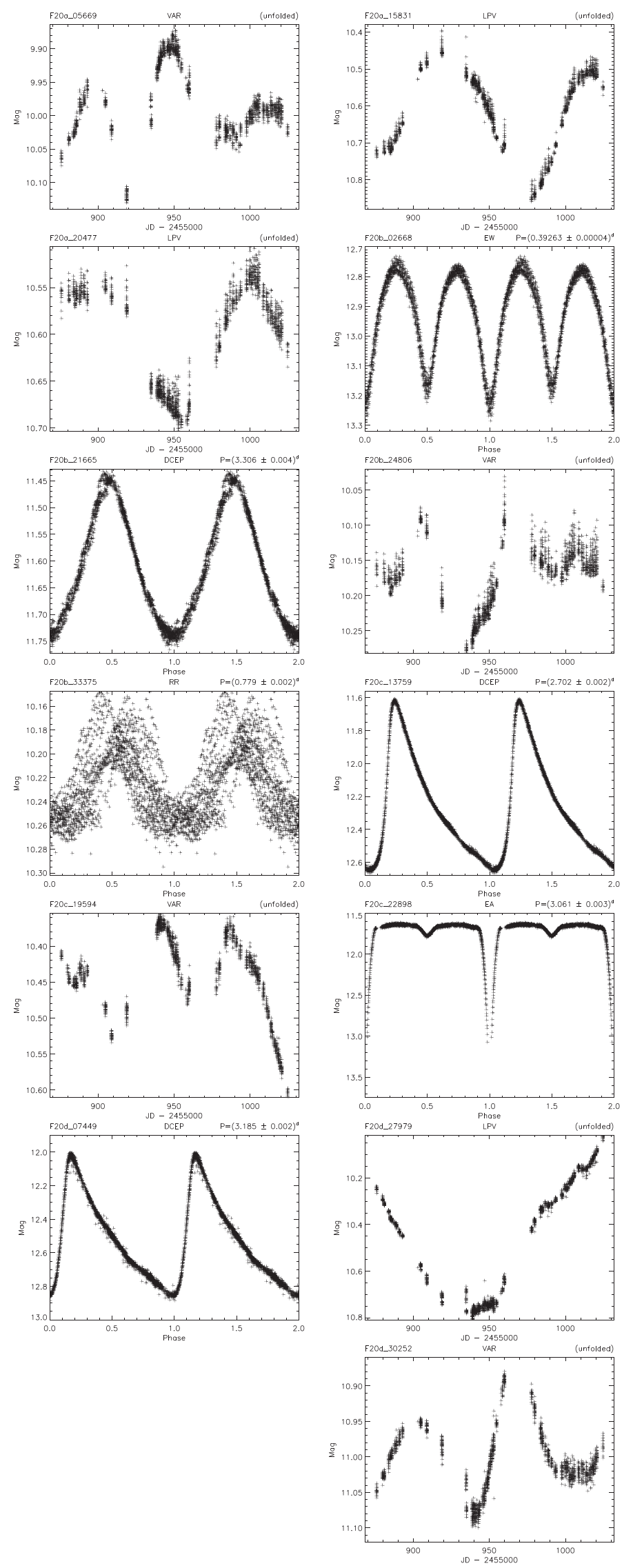
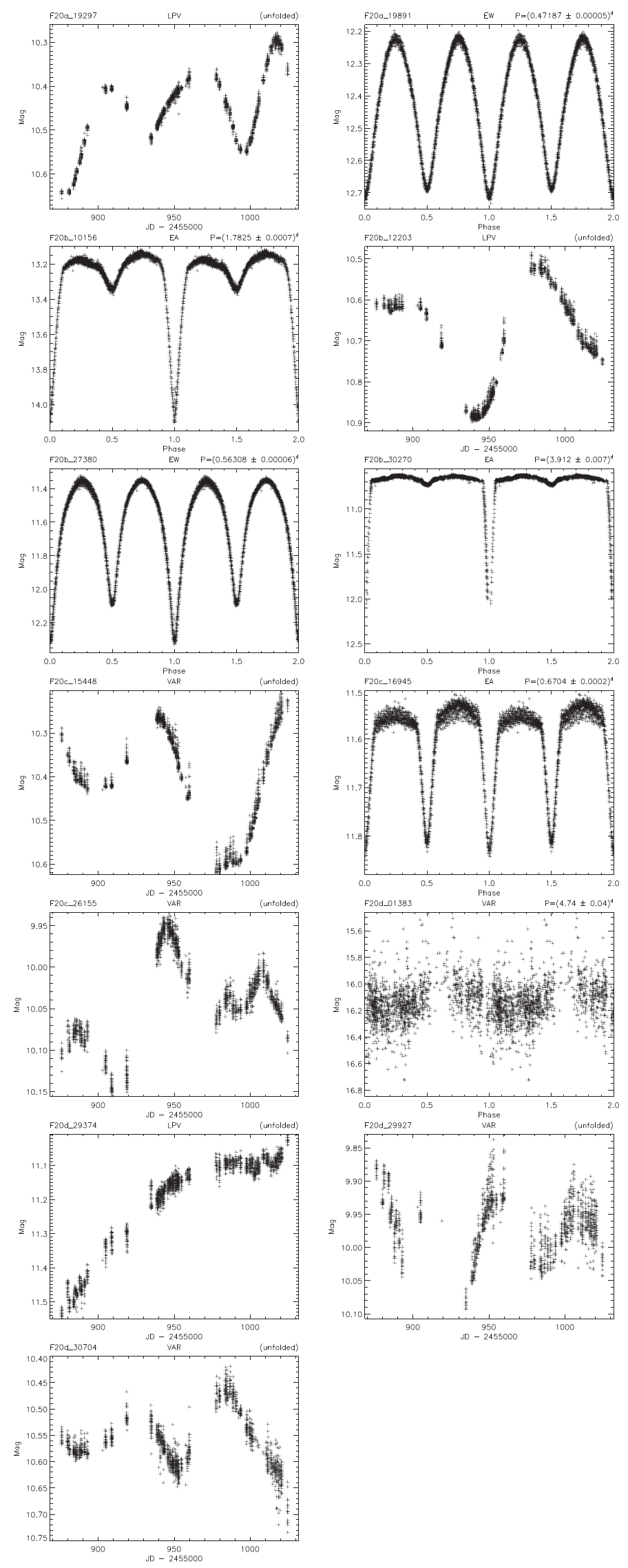

Figure 5. Complete set of light curves of the already known variable stars in the BEST II target field F20. BEST II identifier, variable type, and period (if known) are provided at the top of the figures. 
Table 5

Variable Star Detection Yield in Comparison to Other Surveys

\begin{tabular}{|c|c|c|c|c|c|}
\hline Project & $N_{*}$ & $N_{\text {var }}$ & $N_{\mathrm{var}} / N_{*}$ & Mag & References \\
\hline BEST & 121,811 & 335 & $0.28 \%$ & $11-15$ & $\begin{array}{l}\text { Karoff et al. (2007), Kabath et al. }(2007,2008) \\
\text { Pasternacki et al. (2011) }\end{array}$ \\
\hline BEST II (CoRoT) & 395,818 & 4114 & $1.04 \%$ & $11-17$ & $\begin{array}{l}\text { Kabath et al. (2009a, 2009b), Fruth et al. (2012) } \\
\text { Klagyivik et al. }(2013,2016)\end{array}$ \\
\hline BEST II (F17-F19) & 209,070 & 3040 & $1.45 \%$ & $11-17$ & Fruth et al. (2013) \\
\hline \multicolumn{6}{|c|}{ Other Ground-based Surveys } \\
\hline UNSW & 87,000 & 850 & $0.98 \%$ & $8-14$ & Christiansen et al. (2008) \\
\hline HAT 199 & 98,000 & 1617 & $1.65 \%$ & $8-14$ & Hartman et al. (2004) \\
\hline EROS II & 1913,576 & 1362 & $0.07 \%$ & $11-17$ & Derue et al. (2002) \\
\hline ASAS-2 & 140,000 & 3800 & $2.71 \%$ & $8-13$ & Pojmanski (2000) \\
\hline ASAS-3 & $1.7 \times 10^{7}$ & 50,099 & $0.29 \%$ & $8-14$ & Paczyński et al. (2006) \\
\hline
\end{tabular}

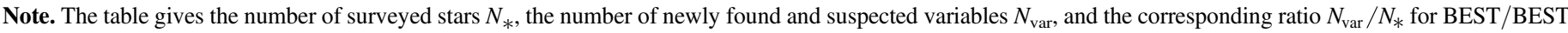

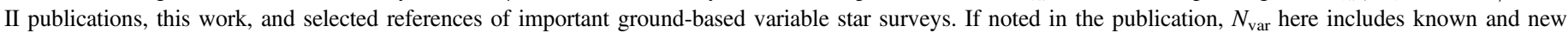
detections, i.e., the whole detection yield of a given survey. The column "Mag" gives each survey's approximate magnitude range.

published Gaia DR2 (Gaia Collaboration et al. 2018a) and highlight our variable stars. The absolute magnitude $M_{G}$ is derived from the Gaia magnitude, the Gaia parallax, and the extinction as reported by Gaia DR2. The color index $\left(G_{\mathrm{BP}}-G_{\mathrm{RP}}\right)_{\text {corr }}$ is determined using the Gaia colors BP, RP, and reddening information, also part of the Gaia catalog. We could calculate the absolute Gaia magnitude for only 30\%$40 \%$ of the BEST II target field F20 objects because of missing extinction information in the Gaia catalog.

Figure 6 shows the resulting BEST II field F20 CDMs for binary systems (upper panel) and pulsating stars (lower panel). In the binary CMD, one can see that binary systems occupy all branches of the diagram as we expect (see Gaia Collaboration et al. 2018b, Figure 4 therein). Their frequency on different branches are according to the expectations (e.g., Eggleton 2006). In the CMD for pulsating variables (lower panel) the $\delta$ Scutis are generally in a good position in the so-called instability strip. Many stars classified as RR Lyrae type stars by period and light-curve shape are actually main-sequence stars according to the Gaia data (see Gaia Collaboration et al. 2018b, Figure 2 therein). Therefore, we think the classification as RR Lyrae based on the photometric information has to be considered carefully. Maybe they have to be classified as spotted stars. According to their position in the CDM, many stars classified as Cepheids are red giants, but Cepheids are actually yellow hyper-giants. These Cepheids might also be reclassified as spotted or rotating stars. In general, we might have difficulties disentangling spotted stars from pulsating stars in our scheme. Finally, one sees a set of LPV and VAR stars with similar absolute magnitude $\left(M_{G} \sim-2 \mathrm{mag}\right)$ and very red colors $\left(\left(G_{\mathrm{BP}}-G_{\mathrm{RP}}\right)_{\text {corr }}>2 \mathrm{mag}\right)$. This may result from the error-affected Gaia color index and/or extinction.

The cross-match with the Gaia DR2 information suggests that our classification is reasonable for eclipsing binaries, but not so reliable for pulsating variables; opposite of the automatic classification routine of Debosscher et al. (2009). However, one should consider that the Gaia DR2 data suffers from systematic errors, in particular, extinction (van Leeuwen et al. 2018). That is why we are limited in achieving our goal of cross-checking our classification and the result must be considered with reservation. A final conclusion can be drawn once the Gaia DR2 information has been validated. We also cannot exclude the possibility that the BEST II - Gaia DR2 cross-identification failed in some cases, since background stars can influence and distort the identification.

\section{Modeling of Binary Systems}

To have a deeper look into the properties of the detected eclipsing binaries and to determine the spot parameters of some of them at the epoch of the observation, eight systems, including the two newly classified binaries NSV 3636 (F20b_027380) and NSV 3640 (F20b_030270), were studied in more detail with the BinaryModel light-curve modeling code developed by Csizmadia et al. (2009). This code is very similar to the Wilson-Devinney Code (Wilson \& Devinney 1971) and it uses a Roche-model to take the proximity effects into account. All details of the modeling not described here are the same as in Klagyivik et al. (2013). The systems for this section were selected on the basis of signal-to-noise $(\mathrm{S} / \mathrm{N})$ ratio, and obviously brighter targets with deeper eclipses produced higher $\mathrm{S} / \mathrm{N}$.

The effective temperatures of the primaries were calculated using the 2MASS $J-K$ color index and they were fixed during the fitting procedure. Systematic errors can be present in these temperatures, owing to unknown reddening. $R$-band linear bolometric and quadratic limb darkening coefficients were chosen from van Hamme (1993) and Claret \& Bloemen (2011), respectively, since this is the closest filter to our white response function. Below $6000 \mathrm{~K}$ stellar temperature, the gravity darkening exponents and Albedos were set to $g=0.32$ and to $A=0.5$, and over it to $g=A=1.0$ (Lucy 1967; Ruciński 1969).

We fitted the mass ratio $Q$, inclination $i$, fill-out factors of the two components $f 1, f 2$ (Mochnacki 1981), effective surface temperature of the secondary star $T 2$, epoch $T_{0}$, height correction $h$ of the maximum brightness at the first quarter 

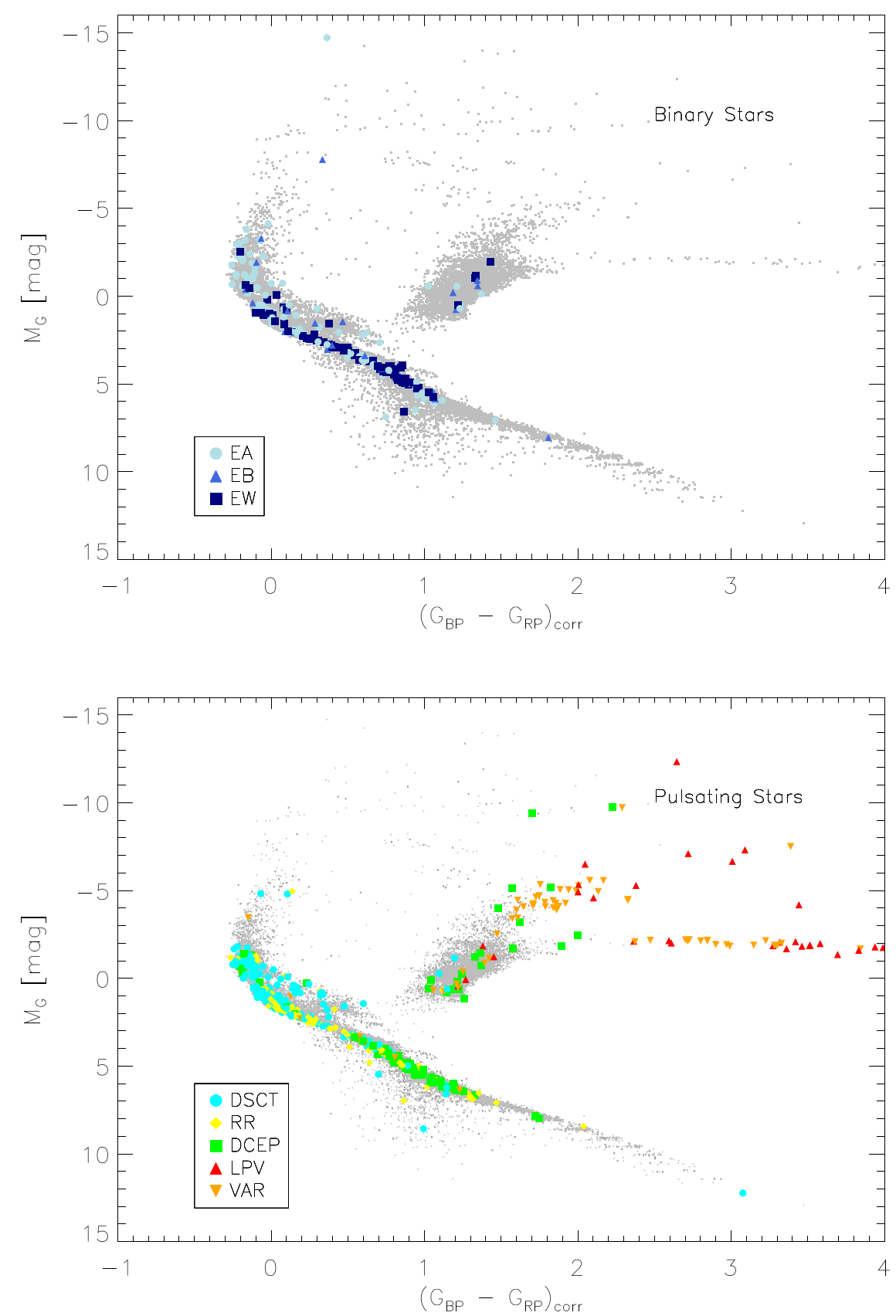

Figure 6. Gaias color-magnitude diagrams of the BEST II target field F20 for binary systems (upper panel) and for pulsating stars (lower panel). The BEST II F20 stars are represented by gray dots, while the colored symbols indicate different variability classes.

refining the normalization of the light curve and stellar spots to one or both of the components if needed. We tried all the possible combinations up to two spots in total (no spot, one spot on the primary star, one spot on the secondary component, etc.). The temperature of a spot is described as the temperature ratio of the spot and the star (temperature factor $=T_{\text {spot }} / T_{\text {star }}$ ). The fits with the smallest $\chi^{2}$ values were accepted and summarized in Table 6, which gives detailed system parameters, and in Figure 7, which shows the resulting fits. Notice that mark ${ }^{\mathrm{a}}$ in Table 6 means that the system was found to be over-contacted when the two fill-out factors are equal to each other. The errors in Table 6 include only modeling uncertainties but no systematic errors of the input parameters.
F20a_003678, F20b_027380, F20c_015786, and F20d_026554 are contact binaries (EW), two of them spotted. We report the spot parameters in Table 6. The spot evolution can be further studied via long-term photometry, and all-sky ground (e.g., PannStarrs, ASAS) and space (e.g., TESS) surveys provide additional data at different epochs, and thus our 2011/12 observations may serve as a baseline extension. The detached binaries (EA) F20a_027661, F20c_001601, and F20d_005502 exhibit stable light curves and probably would be good targets for subsequent radial velocity studies to measure the components' masses precisely. F20b_030270 shows unexplained light-curve variations during primary transit, which deserves further multicolor light-curve studies. 
Table 6

Characteristics of the Modeled Binary Systems

\begin{tabular}{|c|c|c|c|c|c|c|c|c|}
\hline $\begin{array}{l}\text { Catalog ID } \\
\text { BEST II ID } \\
\text { Classification }\end{array}$ & $\begin{array}{c}\text { F20a_003678 } \\
\text { EW }\end{array}$ & $\begin{array}{c}\text { F20a_027661 } \\
\text { EA }\end{array}$ & $\begin{array}{c}\text { NSV } 3636 \\
\text { F20b_027380 } \\
\text { EW }\end{array}$ & $\begin{array}{c}\text { NSV } 3640 \\
\text { F20b_030270 } \\
\text { EA }\end{array}$ & $\begin{array}{c}\text { F20c_001601 } \\
\text { EA }\end{array}$ & $\begin{array}{c}\text { F20c_015786 } \\
\text { EW }\end{array}$ & $\begin{array}{c}\text { F20d_005502 } \\
\text { EA }\end{array}$ & $\begin{array}{c}\text { F20d_026554 } \\
\text { EW }\end{array}$ \\
\hline \multicolumn{9}{|l|}{ Measured Parameters } \\
\hline Magnitude $R_{B}(\mathrm{mag})$ & 15.48 & 14.78 & 11.50 & 10.66 & 13.26 & 13.62 & 13.23 & 13.61 \\
\hline Epoch $T 0$ (HJD-2455,800.0) & 80.372 & 80.325 & 75.830 & 83.035 & 88.463 & 75.841 & 80.759 & 75708 \\
\hline Period $P$ (day) & $0.39492 \pm 0.00008$ & $1.6116 \pm 0.0009$ & $0.56308 \pm 0.00008$ & $3.912 \pm 0.009$ & $1.893 \pm 0.002$ & $0.26821 \pm 0.00004$ & $3.112 \pm 0.005$ & $0.41220 \pm 0.00007$ \\
\hline Amplitude $A$ (mag) & $0.46 \pm 0.05$ & $1.16 \pm 0.05$ & $0.90 \pm 0.08$ & $1.4 \pm 0.4$ & $0.25 \pm 0.02$ & $0.40 \pm 0.04$ & $0.52 \pm 0.05$ & $0.42 \pm 0.04$ \\
\hline \multicolumn{9}{|l|}{ System Parameter } \\
\hline Orbital eccentricity $e$ & \multicolumn{8}{|c|}{0 (circular orbit, fixed) } \\
\hline Temperature $T 1(K)$ & 5388 (fixed) & 4329 (fixed) & 5370 (fixed) & 4414 (fixed) & 2285 (fixed) & 4652 (fixed) & 5731 (fixed) & \\
\hline Mass ratio $Q$ & $0.51 \pm 0.04$ & $4.1 \pm 0.7$ & $1.463 \pm 0.015$ & $5.48 \pm 0.12$ & $1.630 \pm 0.018$ & $5.1 \pm 0.4$ & $6.5 \pm 0.3$ & $4.3 \pm 0.3$ \\
\hline Inclination $\vec{i}\left(^{\circ}\right)$ & $70.0 \pm 0.3$ & $77.3 \pm 1.4$ & $86.6 \pm 0.2$ & $82.9 \pm 0.2$ & $90.0 \pm 0.6$ & $68.0 \pm 0.5$ & $72.16 \pm 0.19$ & $72.3 \pm 0.7$ \\
\hline Fill-out factor $f 1$ & $0.22 \pm 0.04$ & $-1.70 \pm 0.15$ & $0.1087 \pm 0.0008$ & $-3.88 \pm 0.07$ & $-9.75 \pm 0.12$ & $0.2450 \pm 0.0007$ & $-2.00 \pm 0.08$ & $0.06 \pm 0.06$ \\
\hline Fill-out factor $f 2$ & $0.22^{\mathrm{a}}$ & $-4.2 \pm 0.4$ & $0.1087^{\mathrm{a}}$ & $-20.7 \pm 0.5$ & $-1.71 \pm 0.03$ & $0.2450^{\mathrm{a}}$ & $-7.1 \pm 0.3$ & $0.06^{\mathrm{a}}$ \\
\hline Temperature $T 2(K)$ & $5341 \pm 34$ & $3078 \pm 65$ & $5050 \pm 3$ & $2751 \pm 21$ & $2249 \pm 3$ & $4410 \pm 28$ & $3771 \pm 7$ & $3378 \pm 20$ \\
\hline Height correction $h$ & $0.0016 \pm 0.0006$ & $-0.0043 \pm 0.0017$ & $0.00097 \pm 0.00017$ & $-0.00177 \pm 0.00017$ & $-0.0009 \pm 0.0003$ & $-0.0023 \pm 0.0003$ & $0.0038 \pm 0.0003$ & $0.0030 \pm 0.0007$ \\
\hline $\mathcal{X}^{2}$ & 0.506448 & 4.08084 & 1.46310 & 5.47498 & 1.63041 & 5.05016 & 6.47662 & 4.34199 \\
\hline Spot 1 on what star? & $\ldots$ & Primary & Primary & $\ldots$ & $\ldots$ & $\ldots$ & $\ldots$ & Primary \\
\hline Longitude $\lambda 1\left(^{\circ}\right)$ & & $74 \pm 40$ & $149 \pm 59$ & & & & & $114 \pm 20$ \\
\hline Colatitude $\phi 1\left(^{\circ}\right)$ & & $127 \pm 51$ & $302 \pm 16$ & & & & & $99 \pm 10$ \\
\hline Radius ds 1 & & $26 \pm 11$ & $11 \pm 3$ & & & & & $29 \pm 3$ \\
\hline Temperature factor 1 & & $0.95 \pm 0.04$ & $0.6 \pm 0.2$ & & & & & $0.26 \pm 0.03$ \\
\hline Spot 2 on what star? & $\ldots$ & Secondary & Secondary & $\cdots$ & $\cdots$ & $\cdots$ & $\cdots$ & Primary \\
\hline Longitude $\lambda 2\left(^{\circ}\right)$ & & $23 \pm 50$ & $110 \pm 33$ & & & & & $40 \pm 43$ \\
\hline Colatitude $\phi 2\left(^{\circ}\right)$ & & $196 \pm 91$ & $130 \pm 85$ & & & & & $245 \pm 63$ \\
\hline Radius ds2 & & $169 \pm 41$ & $4 \pm 44$ & & & & & $54 \pm 43$ \\
\hline Temperature factor 2 & & $0.96 \pm 0.30$ & $0.6 \pm 0.3$ & & & & & $1.02 \pm 0.07$ \\
\hline \multicolumn{9}{|l|}{ Stellar Fractional Radii } \\
\hline \multicolumn{9}{|l|}{ Primary } \\
\hline R1 (pole) & $0.424 \pm 0.004$ & $0.1968 \pm 0.0019$ & $0.331 \pm 0.003$ & $0.1468 \pm 0.0015$ & $0.1139 \pm 0.0012$ & $0.240 \pm 0.003$ & $0.1704 \pm 0.0017$ & $0.245 \pm 0.003$ \\
\hline R1 (side) & $0.486 \pm 0.005$ & $0.211 \pm 0.002$ & $0.383 \pm 0.004$ & $0.1522 \pm 0.0015$ & $0.1145 \pm 0.0012$ & $0.292 \pm 0.003$ & $0.1829 \pm 0.0019$ & $0.289 \pm 0.003$ \\
\hline R1 (back) & $0.453 \pm 0.005$ & $0.201 \pm 0.002$ & $0.348 \pm 0.004$ & $0.1483 \pm 0.0015$ & $0.1142 \pm 0.0012$ & $0.251 \pm 0.003$ & $0.1737 \pm 0.0018$ & $0.255 \pm 0.003$ \\
\hline R1 (point) & & $0.215 \pm 0.002$ & & $0.1532 \pm 0.0015$ & $0.1146 \pm 0.0012$ & & $0.1862 \pm 0.0019$ & \\
\hline \multicolumn{9}{|l|}{ Secondary } \\
\hline R2 (pole) & $0.197 \pm 0.002$ & $0.334 \pm 0.003$ & $0.315 \pm 0.003$ & $0.217 \pm 0.002$ & $0.270 \pm 0.003$ & $0.455 \pm 0.005$ & $0.355 \pm 0.004$ & $0.435 \pm 0.004$ \\
\hline R2 (side) & $0.197 \pm 0.002$ & $0.334 \pm 0.003$ & $0.315 \pm 0.003$ & $0.217 \pm 0.002$ & $0.270 \pm 0.003$ & $0.455 \pm 0.005$ & $0.355 \pm 0.004$ & $0.435 \pm 0.004$ \\
\hline R2 (back) & $0.197 \pm 0.002$ & $0.334 \pm 0.003$ & $0.315 \pm 0.003$ & $0.217 \pm 0.002$ & $0.270 \pm 0.003$ & $0.455 \pm 0.005$ & $0.355 \pm 0.004$ & $0.435 \pm 0.004$ \\
\hline R2 (point) & & $0.334 \pm 0.003$ & & $0.217 \pm 0.002$ & $0.270 \pm 0.003$ & & $0.355 \pm 0.004$ & \\
\hline
\end{tabular}

Note.

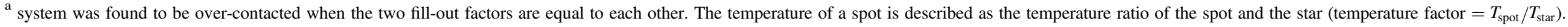



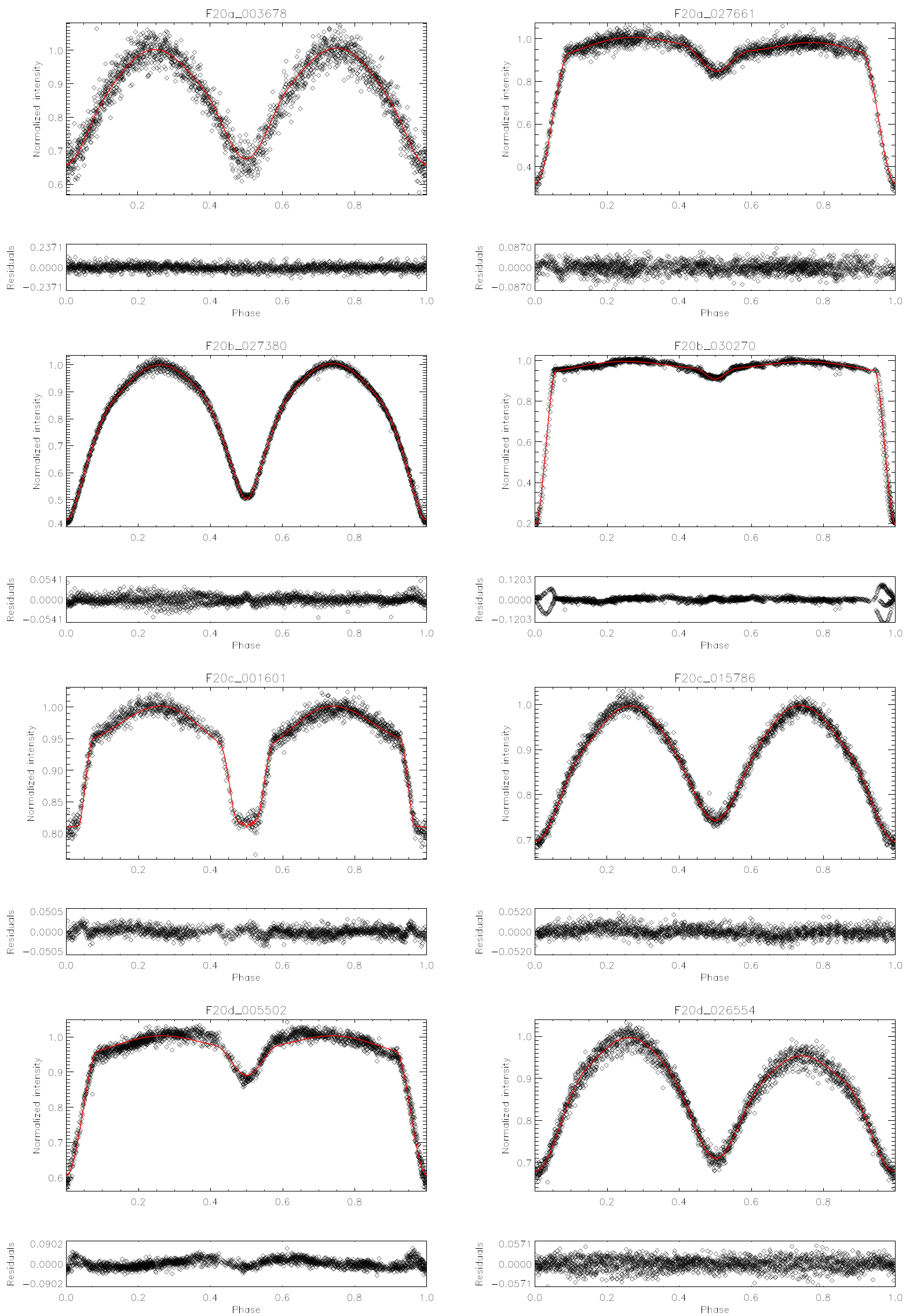

Figure 7. Light-curve modeling results of eight binary systems in the BEST II target field F20. Points represent the observation and the red solid line denotes the fit to it. The residuals are shown in the lower panel. The classified, previously known varitable stars NSC 3636 and NSC 3640 are shown in the second upper panel. 


\section{Summary}

The BEST II telescope was used to search for stellar light variations within the Puppis (Pup) constellation close to the Galactic plane. Data collected in 61 nights between 2011 November 9 and 2012 April 6 were processed and comprehensively analyzed to classify all variable stars in the observed target field. We identified 2169 variable stars, including 1829 new, 26 previously known, and 314 suspected stars determining epoch, period, amplitude, and variability class. For the two previously known but unclassified variables NSV 3636 and NSV 3640, we were able to provide periods and classification as eclipsing binaries.

To verify the variable classification of our BEST II target field F20, we cross-matched it with the recently published Gaia DR2 information by producing a CDM wherein the different variability classes populate well-defined regions. This crossmatch indicates that our classification is reasonable for eclipsing binaries, and less reliable for pulsating variables. However, no reliable statements are possible because of missing or systematic error-affected Gaia data. But, in general, it emphasizes that a classification of variable stars combining both photometric and astrometric information increases its accuracy.

In addition, we presented the light-curve modeling results for eight eclipsing binary systems, including NSV 3636 and NSV 3640.

Our variable star census is fully available in an online catalog that can be used for further studies. With the results reported here, the search for light variation by the BEST/BEST II telescopes has been completed and the project closed.

We thank Thomas Pasternacki for his efforts during the BEST II Field F20 observations. Sz.Cs. acknowledges participation to the Hungarian OTKA Grant K113117.

This work has made use of the 2MASS, GCVS catalog, the AAVSO variable star search index (VSX), and the data from the European Space Agency (ESA) mission Gaia, processed by the Gaia Data Processing and Analysis Consortium (DPAC). Funding for the DPAC has been provided by national institutions, in particular, the institutions participating in the Gaia Multilateral Agreement.

\section{ORCID iDs}

\section{J. Cabrera @ (ttps://orcid.org/0000-0001-6653-5487}

\section{References}

Baglin, A., Auvergne, M., Barge, P., et al. 2006, in ESA Special Publication 1306, The CoRoT Mission Pre-Launch Status-Stellar Seismology and Planet Finding, ed. M. Fridlund et al. (Paris: ESA), 33

Christiansen, J. L., Derekas, A., Kiss, L. L., et al. 2008, MNRAS, 385, 1749

Claret, A., \& Bloemen, S. 2011, A\&A, 529, A75

Csizmadia, S., Borkovits, T., Paragi, Z., et al. 2009, ApJ, 705, 436

Csizmadia, S., Moutou, C., Deleuil, M., et al. 2011, A\&A, 531, A41
Debosscher, J., Sarro, L. M., López, M., et al. 2009, A\&A, 506, 519

Deeg, H. J., Gillon, M., Shporer, A., et al. 2009, A\&A, 506, 343

Derue, F., Marquette, J.-B., Lupone, S., et al. 2002, A\&A, 389, 149

Eggleton, P. 2006, Evolutionary Processes in Binary and Multiple Stars (Cambridge: Cambridge Univ. Press)

Feast, M., \& Whitelock, P. A. 2014, IAU Symp. 298, Setting the scene for Gaia and LAMOST, ed. S. Feltzing, (Cambridge: Cambridge Univ. Press), 40

Fruth, T., Cabrera, J., Chini, R., et al. 2013, AJ, 146, 136

Fruth, T., Kabath, P., Cabrera, J., et al. 2012, AJ, 143, 140

Gaia Collaboration, Brown, A. G. A., Vallenari, A., et al. 2018a, A\&A, 616, A1

Gaia Collaboration, Eyer, L., Rimoldini, L., et al. 2018b, arXiv:1804.09382

Gaia Collaboration, Prusti, T., de Bruijne, J. H. J., et al. 2016, A\&A, 595, A1

Hartman, J. D., Bakos, G., Stanek, K. Z., \& Noyes, R. W. 2004, AJ, 128, 1761

Henden, A. A., Templeton, M., Terrell, D., et al. 2016, yCat, 2336, 0

Hilditch, R. W. 2004, in ASP Conf. Ser. 318, Spectroscopically and Spatially Resolving the Components of the Close Binary Stars, ed. R. W. Hilditch,

H. Hensberge, \& K. Pavlovski (San Francisco, CA: ASP), 3

Hubble, E. P. 1925, ApJ, 62, 409

Kabath, P., Eigmüller, P., Erikson, A., et al. 2007, AJ, 134, 1560

Kabath, P., Eigmüller, P., Erikson, A., et al. 2008, AJ, 136, 654

Kabath, P., Erikson, A., Rauer, H., et al. 2009a, A\&A, 506, 569

Kabath, P., Fruth, T., Rauer, H., et al. 2009b, AJ, 137, 3911

Karoff, C., Rauer, H., Erikson, A., et al. 2007, AJ, 134, 766

Klagyivik, P., Csizmadia, S., Pasternacki, T., et al. 2013, ApJ, 773, 54

Klagyivik, P., Csizmadia, S., Pasternacki, T., et al. 2016, AJ, 151, 110

Lucy, L. B. 1967, AJ, 72, 309

Mel'nik, A. M., Rautiainen, P., Berdnikov, L. N., Dambis, A. K., \& Rastorguev, A. S. 2015, AN, 336, 70

Mochnacki, S. W. 1981, ApJ, 245, 650

Paczyński, B., Szczygieł, D. M., Pilecki, B., \& Pojmański, G. 2006, MNRAS, 368, 1311

Pasternacki, T., Csizmadia, S., Cabrera, J., et al. 2011, AJ, 142, 114

Pietrukowicz, P., Dziembowski, W. A., Mróz, P., et al. 2013, AcA, 63, 379

Pojmanski, G. 2000, AcA, 50, 177

Pojmanski, G. 2002, AcA, 52, 397

Poretti, E., Le Borgne, J. F., Rainer, M., et al. 2015, MNRAS, 454, 849

Rauer, H., Eislöffel, J., Erikson, A., et al. 2004, PASP, 116, 38

Rauer, H., Erikson, A., Kabath, P., et al. 2010, AJ, 139, 53

Rauer, H., Fruth, T., \& Erikson, A. 2008, PASP, 120, 852

Ruciński, S. M. 1969, AcA, 19, 245

Samus, N. N., Kazarovets, E. V., Durlevich, O. V., Kireeva, N. N., \& Pastukhova, E. N. 2017, ARep, 61, 80

Schwarzenberg-Czerny, A. 1996, ApJL, 460, L107

Skiff, X. 2004, yCat, 3233, 0

Skiff, B. A. 2014, yCat, 2023, 1

Soszyński, I., Poleski, R., Udalski, A., et al. 2008, AcA, 58, 163

Soszyński, I., Udalski, A., Pietrukowicz, P., et al. 2011, AcA, 61, 285

Southworth, J. 2012, in Orbital Couples: Pas de Deux in the Solar System and the Milky Way, ed. F. Arenou \& D. Hestroffer (Paris: Observatorie de Paris), 51

Stetson, P. B. 1996, PASP, 108, 851

Strohmeier, W., \& Knigge, R. 1975, VeBam, 10, 116

Strohmeier, W., Knigge, R., \& Ott, H. 1964, IBVS, 70, 1

Takamizawa, K. 2000, http://www.kusastro.kyoto-u.ac.jp/vsnet/information. html

van Hamme, W. 1993, AJ, 106, 2096

van Hoof, A. 1941, C\&T, 57, 321

van Leeuwen, F., de Bruijne, J. H. J., Arenou, F., et al. 2018, Gaia DR2 Documentation, Tech. Rep., GDR2

Watson, C., Henden, A. A., \& Price, A. 2015, yCat, 1020, 27

Wilson, R. E., \& Devinney, E. J. 1971, ApJ, 166, 605

Zebrun, K., Soszynski, I., Wozniak, P. R., et al. 2001, AcA, 51, 317

Zhang, X.-B., Deng, L.-C., Xin, Y., \& Zhou, X. 2003, ChJAA, 3, 151 